\title{
Antimicrobial Susceptibility Pattern of Escherichia coli Isolated from Urinary Tract Infected Patients Attending Bir Hospital
}

\author{
Amit Raj Sharma ${ }^{1}$, Dwij Raj Bhatta ${ }^{1}$, Jyotsna Shrestha ${ }^{2}$ and Megha Raj Banjara ${ }^{1}$ \\ ${ }^{1}$ Central Department of Microbiology, Tribhuvan University, Kathmandu \\ ${ }^{2}$ Pathology Department, Bir Hospital, Kathmandu \\ e-mail: amritrsharma@gmail.com
}

\begin{abstract}
Antibiotic resistance among uropathogens is emerging public health problem. This study was done for assessing antibiotic and multidrug resistance (MDR) patterns of Escherichia coli at Bir Hospital, Kathmandu, among suspected urinary tract infection (UTI) patients from January to March, 2011. Altogether, 739 urine samples were analysed by semi-quantitative culture method and uropathogens were identified by conventional methods. E. coli was tested (109 samples) for antimicrobial susceptibility by Kirby Bauer disc diffusion method as per Clinical and Laboratory Standard Institute (CLSI) guidelines. Out of 739 samples, 27.3\% gave significant growth while 3.1\% and 29.2\% samples gave mixed and non-significant growth respectively. E. coli was found to be most predominant isolate (54.0\%) followed by coagulase negative Staphylococci (CoNS) (21.3\%) and Enterococcus spp. (7.3\%). Nitrofurantoin was found to be the most effective antibiotic followed by ciprofloxacin and ofloxacin while cephalexin was least effective. Out of 109 E. coli isolates, 90.8\% were MDR strains and most of the isolates had a very high multiple antibiotic resistance (MAR) index, suggesting the origin of the isolates to be of high antibiotic usage. E. coli showed higher rate of resistance towards commonly used oral antibiotics. However, nitrofurantoin is still active against organisms. Thus, nitrofurantoin could be the choice for empirical therapy of UTI.
\end{abstract}

Key words: Escherichia coli, multidrug resistance, multiple antibiotic resistance index, urinary tract infection

\section{Introduction}

Urinary tract infections (UTIs) are one of the most common infectious diseases ranking next to upper respiratory tract infection. UTIs are often associated with significant morbidity and mortality (Ramesh et al. 2008). Worldwide, about 150 million people are diagnosed with urinary tract infection each year, costing the global economy in excess of 6 billion dollars (Gonzalez \& Schaeffer 1999). E. coli, the most common member of the family Enterobacteriaceae, accounts for $75.0-90.0 \%$ of all UTIs in both inpatients and outpatients (Dromigny et al. 2005). E. coli present in the gastrointestinal tract as a commensal provide the pool for initiation of UTI and certain serotypes of $E$. coli responsible for uropathogenicity were traditionally designated as uropathogenic E. coli (UPEC) (Raksha et al. 2003). UTI is a common disease ailment among Nepalese population as well as one of the commonest nosocomial infection (Kattel et al. 2008). According to the annual report of fiscal year (2010/2011) published by the Department of Health Services, morbidity of UTI among outpatients were 265,143 . The present study was conducted to determine the prevalence and antibiotic susceptibility pattern of $E$. coli associated urinary tract infection among inpatients and outpatients.

\section{Methodology}

In a descriptive cross sectional study conducted from January to March 2011, a total of 739 urine samples from suspected UTI patients visiting Bir Hospital, Kathmandu were processed in the microbiology laboratory of this hospital for the isolation of E. coli. Each sample was mixed well and aseptically inoculated with $4 \mathrm{~mm}$ diameter nichrome wire loop on blood agar and MacConkey agar plates and incubated at $37^{\circ} \mathrm{C}$ for 24 hours aerobically. Significant UTI was defined as the presence of $>10^{5}$ colony forming unit $(\mathrm{CFU}) / \mathrm{ml}$ in the culture. All positive cultures were further identified by their cultural characteristics, Gram stain and battery of biochemical reaction. E. coli was identified on the 
basis of triple sugar iron agar (TSI), sulphide indole motility (SIM) test medium, citrate utilization and urease production. The antimicrobial susceptibility testing (AST) of E. coli isolates was done by Kirby Bauer Disc Diffusion Method as per CLSI guideline (CLSI 2011). The antibiotic discs of HiMedia (India) used were ampicillin $(10 \mu \mathrm{g})$, cephalexin $(30 \mu \mathrm{g})$, nalidixic acid (30 $\mu$ g), ciprofloxacin (5 $\mu \mathrm{g})$, ofloxacin (5 $\mu \mathrm{g})$, norfloxacin $(10 \mu \mathrm{g})$, nitrofurantoin $(300 \mu \mathrm{g})$ and co-trimoxazole (1.25/23.75 $\mu \mathrm{g})$. A reference strain, $E$. coli ATCC 25922 was used as control. Multiple antibiotic resistance (MAR) index was determined using the formula MAR=x/y, where $\mathrm{x}$ was number of antibiotics to which test isolate displayed resistance and $y$ is the total number of antibiotics to which the test organism has been evaluated for sensitivity (Akinjogunla \& Enabulele 2010). Data collected were analysed by using PASW (Predictive Analytical Software), version 18.0, the premier vendor for (Statistical Package for the Social Sciences) program. A p-value of less than or equal to 0.05 was considered to be statistically significant $(\mathrm{p} \leq 0.05)$.

\section{Results and Discussion}

A total of 739 urine samples, 202 (27.3\%) samples showed significant growth, whereas majority of the samples i.e. 298 (40.3\%) showed no growth, 216 showed non-significant growth, and out of the total only 23 samples showed mixed growths. E. coli (54.0\%) was the most predominant uropathogen followed by CoNS (21.3\%), Enterococcus spp. (7.3\%), Pseudomonas aeruginosa (4.0\%) and Klebsiella spp. (2.7\%). Acinetobacter spp. and Candida spp. were $2.2 \%$ each. Other bacterial isolates were $1.0 \%$ or less than $1.0 \%$. The distribution of $E$. coli were found to be the most frequent in age group 16-49 years in both sexes i.e. 13 and 60 in male and female respectively. In age group 1-15 years only two $E$. coli were isolated from females. The sexwise distribution showed that females $(65.3 \%)$ were more susceptible to UTI than males (34.7\%).

E. coli, the most common uropthogen isolated more commonly from the female patients comparatively to the male patients and isolation of E. coli among female patients is statistically significant $(\mathrm{p}<0.05)$ whereas, isolate of CoNS was more common among male patient; however, isolation of CoNS more among male patients is statistically insignificant (p>0.05) (Table 1).

Table 1. Distribution of uropathogens among males and females

\begin{tabular}{l|c|c|c}
\multicolumn{1}{c|}{ Uropathogens } & Male & Female & p- value \\
\hline Escherichia coli & $24(34.3)$ & $85(64.4)$ & 0.0001 \\
Coagulase negative Staphylococcus & $22(31.4)$ & $21(15.9)$ & 0.084 \\
(CoNS) & $9(12.9)$ & $7(5.3)$ & 0.145 \\
Enterococcus spp. & $4(5.7)$ & $6(4.5)$ & 1.0 \\
Pseudomonas aeruginosa & $1(1.4)$ & $4(3.0)$ & 0.654 \\
Acinetobacter spp. & $2(2.9)$ & $3(2.3)$ & 1.0 \\
Candida spp. & $3(4.3)$ & $3(2.3)$ & 0.682 \\
Klebsiella spp. & $2(2.9)$ & $2(1.5)$ & 0.643 \\
Staphylococcus aureus & $2(2.9)$ & $0(0.0)$ & 0.149 \\
Citrobacter spp. & $0(0.0)$ & $1(0.8)$ & 1.0 \\
Enterobacter spp. & $1(1.4)$ & $0(0.0)$ & 0.387 \\
Salmonella Typhi & $\mathbf{7 0 ( 1 0 0 . 0 )}$ & $\mathbf{1 3 2 ( 1 0 0 . 0 )}$ & \\
\hline \multicolumn{1}{c|}{ Total } & &
\end{tabular}

Majority of E. coli showed susceptibility towards nitrofurantoin (94.5\%) followed by ciprofloxacin and ofloxacin with the susceptibility of $50.5 \%$ for each drug. Cephalexin (7.3\%) was found least effective drug followed by ampicillin (18.3\%). Norfloxacin, nalidixic acid and co-trimoxazole were found effective only for less than half of the isolates of $E$. coli (Table 2).
Table 2. Antibiotic susceptibility pattern of $E$. coli

\begin{tabular}{l|c|c}
\hline Antibiotics & Sensitive & Resistant \\
\hline Ampicillin & $20(18.3)$ & $89(81.7)$ \\
Cephalixin & $8(7.3)$ & $101(92.7)$ \\
Nalidixic acid & $23(21.1)$ & $86(78.9)$ \\
Ciprofloxacin & $55(50.5)$ & $54(49.5)$ \\
Ofloxacin & $55(50.5)$ & $54(49.5)$ \\
Norfloxacin & $51(46.8)$ & $58(53.2)$ \\
Co-trimoxazole & $50(45.9)$ & $59(54.1)$ \\
Nitrofurantoin & $103(94.5)$ & $6(5.5)$ \\
\hline
\end{tabular}


Amit Raj Sharma et al./Antimicrobial Susceptibility Pattern.

Taking resistant to two or more classes of antibiotics as MDR, it was detected in $90.8 \%$ isolates. Among the

Table 3. Multidrug resistance (MDR) pattern of $E$. coli
99 (90.8\%) MDR strains, 21(19.3\%) were resistant to two antibiotics and 78 (71.6\%) were resistant to three or more antibiotics (Table 3).

\begin{tabular}{|c|c|c|c|c|c|c|c|}
\hline & & & \multicolumn{4}{|c|}{ Resistance to antibiotic } & \multirow[b]{3}{*}{$\begin{array}{c}\text { Percen } \\
90.8\end{array}$} \\
\hline Bacterial isolate & No. of isolates & & MDR S & & & & \\
\hline Escherichia coli & 109 & $\begin{array}{c}0 \text { Drug } \\
0\end{array}$ & $\begin{array}{c}1 \text { Drug } \\
10\end{array}$ & $\begin{array}{c}2 \text { Drug } \\
21\end{array}$ & $\begin{array}{c}>2 \text { Drug } \\
78\end{array}$ & 99 & \\
\hline
\end{tabular}

Sixteen multidrug resistance patterns were observed in E. coli for the eight antimicrobial agents tested. Resistance to Amp-Na-Cp-Cip-Of-Nx-Nit was the most frequent pattern observed in $30.3 \%$ of $E$. coli isolates, whereas Na-Cp-Cot, Amp-Na-Cp-Nx, Amp-Na-Cp-CipNx, Na-Cip-Of-Nx-Cot and Amp-Na-Cp-Cip-Of-Nx-Nit were the least frequent pattern observed in $1.0 \%$ of $E$. coli for each (Table 4).

Table 4. Antibiotic resistance pattern of MDR E. coli strains

\begin{tabular}{|c|c|}
\hline Antibiotic resistant pattern & Number (\%) \\
\hline Amp-Сp & 13(13.1) \\
\hline $\mathrm{Na}-\mathrm{Cp}$ & $4(4.0)$ \\
\hline Na-Cot & $4(4.0)$ \\
\hline Amp-Cp-Cot & $2(2.0)$ \\
\hline Amp-Na-Cp & $5(5.1)$ \\
\hline Na-Cp-Cot & $1(1.0)$ \\
\hline Amp-Na-Cp-Nx & $1(1.0)$ \\
\hline Amp-Na-Cp-Cot & $12(12.1)$ \\
\hline Amp-Na-Cp-Nx-Cot & $2(2.0)$ \\
\hline Amp-Na-Cp-Cip-Nx & $1(1.0)$ \\
\hline Na-Cip-Of-Nx-Cot & $1(1.0)$ \\
\hline Amp-Na-Cp-Cip-Of-Nx & $15(15.2)$ \\
\hline Amp-Na-Cp-Of-Nx-Cot & $2(2.0)$ \\
\hline Amp-Na-Cp-Cip-Of-Nx-Cot & $30(30.3)$ \\
\hline Amp-Na-Cp-Cip-Of-Nx-Nit & $1(1.0)$ \\
\hline Amp-Na-Cp-Cip-Of-Nx-Nit-Cot & $5(5.1)$ \\
\hline Total MDR isolates & 99(100.0) \\
\hline
\end{tabular}

Note: Amp-Ampicillin, Cp-Cephalexin, NaNalidixic acid, Cot-Co-trimoxazole, $\mathrm{Nx}$ Norfloxacin, Cip-Ciprofloxacin, OfOfloxacin, Nit-Nitrofurantoin

Out of 109 E. coli isolates, only 10 showed MAR index of 0.1 i.e. these isolates were only resistant to one antibiotic. However, five isolates showed MAR index of 1 i.e. these isolates were resistant to all the antibiotics used in antibiotic susceptibility testing (Table 5).
Table 5. Multiple antibiotic resistance (MAR) indices of $E$. coli

\begin{tabular}{l|c}
\hline MAR index & $\begin{array}{c}\text { Frequency of MAR } \\
\text { index } \\
\text { E. coli }(\mathbf{n}=\mathbf{1 0 9})\end{array}$ \\
\hline 0 & $0(0.0)$ \\
0.1 & $10(9.2)$ \\
0.2 & $21(19.3)$ \\
0.3 & $8(7.3)$ \\
0.4 & $0(0.0)$ \\
0.5 & $13(11.9)$ \\
0.6 & $4(3.7)$ \\
0.7 & $17(15.6)$ \\
0.8 & $31(28.4)$ \\
0.9 & $0(0.0)$ \\
1.0 & $5(4.6)$ \\
\hline
\end{tabular}

Our study was conducted among outpatients and inpatients suspected of urinary tract infection, attending Bir Hospital, Kathmandu. In this study, 27.3\% urine specimens from suspected UTI patients gave significant growth. The results are in agreement with other investigators from Nepal (Chhetri et al. 2001, Karki et al. 2004, Kumari et al. 2005, Rai et al. 2008, Acharya et al. 2011) and rest of the world (Levitt 1993, Bashar et al. 2009). The majority of urine specimens showed no growth (40.3\%). This may be due to patients undergoing antibiotics therapy which has inhibited or destroyed the bacterial growth (Okonofua \& Okonofu 1989), or slow growing organisms, and which were not able to grow on the routine culture media used (Kattel et al. 2008). This study showed that the commonest isolates were E. coli (54\%), CoNS (21.3\%), Enterococcus spp. (7.3\%), Pseudomonas aeruginosa (4.0\%) and Klebsiella spp. (2.7\%). In a study from Kathmandu, in combined group, outpatients as well as inpatients, E. coli (59.6\%) was the commonest one followed by Staphylococcus aureus (12.5\%), Klebsiella spp. (10.8), Enterococcus spp. (7.9\%) and Pseudomonas aeruginosa (5.0\%) (Kattel et al. 2008). $E$. coli was isolated as the most predominant isolate and accounted for $54.0 \%$ of the total uropathogens 
and agreed with other studies (Chhetri et al. 2001, Dhakal et al. 2002, Jha \& Bapat 2005, Das et al. 2006, Kattel et al. 2008) in Nepal. The results are also agreed with the study done at international context, which indicated that the Gram negative bacteria mostly $E$. coli was the commonest one isolated in patients with UTI (Tambekar et al. 2006, Ahmed \& Imran 2008, Biadglegne \& Abera 2009, Omigie et al. 2009, Manjunath et al. 2011, Alzohairy \& Khadri 2011).

E. coli, the most common uropthogen isolated more commonly from female patients comparatively to the male patients and isolation of $E$. coli more among female patients is statistically significant $(\mathrm{p}<0.05)$ whereas, isolate of CoNS was more common among male patient; however, isolation of CoNS more among male patients is statistically insignificant ( $\mathrm{p}>0.05)$. The reason for the higher prevalence of CoNS in males is not clear, though lack of circumcision, receptive anal intercourse and HIV infection recognised as risk factor for males (Orrett \& Davis 2006). The study revealed that females (65.3\%) were more susceptible to UTI than males (34.7\%), which is also similar to other studies (Dimitrov et al. 2003, Akram et al. 2007, Arjunan et al. 2010, Manjunath et al. 2011, Alzohairy \& Khadri 2011, Acharya et al. 2011). The increased incidence of the urinary tract infection in women is conditioned by favouring anatomic factors, by hormonal changes and by the urodynamic disturbance occurring with age (Bobos et al. 2010).

Most E. coli isolates in our study were resistant to ampicillin (81.7\%) which resembles other studies (Rashedmarandi et al. 2008, Behroozi et al. 2010, Farshad et al. 2010a, Farshad et al. 2011). Overall quinolone resistance of $E$. coli were $57.7 \%$. Increased resistance in quinolones against $E$. coli may reflect the overuse of these drugs for the treatment of UTI (Saleh et al. 2009). Another factor could be the generalized use of fluroquinolones in animals feed (especially in poultry), and the subsequent transmission of resistant to strains from animals to humans (Miller \& Tang 2004). In our study, only 5.5\% isolates were resistant to nitrofurantoin. These findings are in agreement in with other workers (Moniri et al. 2003, Bean et al. 2008, Biadglegne \& Abera 2009, Raza et al. 2011, Bahadin et al. 2011, Baral et al. 2012). Nitrofurantoin was also found as the most effective antimicrobial in UTI caused by E. coli from studies in Nepal (Karki et al. 2004, Shrestha et al. 2007, Sharma et al. 2011, Rijal et al. 2012) and rest of the world (Sahm et al. 2001, Jamie et al. 2002, Alos et al. 2004, Okonko et al. 2009, Behroozi et al. 2010, Eryilmaz et al. 2010). However, Akram et al. (2007), Kausar et al. (2009), and Arjunan et al. (2010) were reported higher rate of $E$. coli resistance to nitrofurantoin. In case of the present study, nitrofurantoin was found to be the most effective antimicrobial. Resistance to nitrofurantoin among $E$. coli isolates from UTIs remained low despite of more than 50 year's widespread use of the drug (Kahlmeter 2000, Mazzulli et al. 2001). Reason for the lack of emerging resistance are not fully understood, but likely include restricting use to indication for urinary infection, limited systemic absorption, and the need for multiple genetic mutations for the bacteria to develop resistance (Nicolle et al. 2006). Most of the $E$. coli isolates showed the multidrug resistant (90.8\%) in agreement with other studies that found multidrug resistant $E$. coli ranging from 67.0 to $100.0 \%$ (Bashar et al. 2009, Moyo et al. 2010, Farshad et al. 2010a, Hassan et al. 2011). The higher MDR may be due to large portion of $E$. coli isolates being previously exposed to several antibiotics. In this study the antimicrobial agents showed that E. coli were highly resistant to commonly used antibiotics i.e. cephalexin, amipicllin, nalidixic acid, ciprofloxacin, ofloxacin, norfloxacin, and co-trimoxazole. The resistance rate of E. coli to commonly used antibiotics was: ampicillin (81.7\%), cephalexin (92.7\%), nalidixic acid (78.9\%), ciprofloxacin (49.5\%), ofloxacin (49.5\%), norfloxacin (53.2\%), co-trimoxazole (54.1\%) and nitrofurantoin (5.5\%). The increasing resistance of co-trimoxazole to $E$. coli has been reported in other studies from Nepal and other countries (Kattel et al. 2008, Rai et al. 2008, Bashar et al. 2009, Biadglegne \& Abera 2009, AbouDobara et al. 2010, Jadhav et al. 2011). They were higher than the rate reported in our study. Ampicillin resistance among $E$. coli was $81.7 \%$, which is comparable with other studies (Rafique et al. 2002, Biadglegne \& Abera 2009, Beheroozi et al. 2011, Alzohairy \& Khadri 2011). On the basis of our finding, antimicrobials such as ampicillin and co-trimoxazole should no longer be recommended for initial empirical therapies for UTIs.

Multiple antibiotic resistance (MAR) index is a tool that reveals the spread of bacterial resistance in a given population (Krumpermann 1983). An MAR index greater than 0.2 implies that the strains of such bacteria originate from an environment where several 
antibiotics are used (Ehinmidu 2003). The MAR indices of $E$. coli obtained in this study is a possible indication that a very large proportion of the bacterial isolates have been exposed to several antibiotics.

Higher resistance rate to all antibiotics used in this study except nitrofurantoin may be explained by high and uncontrolled use of these antibiotics in our institutions.

Most E. coli isolates are highly resistant to commonly prescribed antibiotics (ampicillin, cephalexin, quinolones and co-trimoxazole), but are still susceptible to nitrofurantoin which should be considered as preferred therapeutic agent once the organism is identified.

\section{Acknowledgements}

We greatly acknowledge the support provided by Bir Hospital and the Central Department of Microbiology, Tribhuvan University for conducting this research.

\section{References}

Abou-Dobara, M.I., M.A. Deyab, E.M. Elsawy and H.H. Mohamed. 2010. Antibiotic susceptibility and genotype patterns of Escherichia coli, Klebsiella pneumonia and Pseudomonas aeruginosa isolated from urinary tract infected patients. Pol. J. Microbiol. 59 (3): 207-212.

Acharya, A., R. Gautam and L. Subedee. 2011. Uropathogens and their antimicrobial susceptibility pattern in Bharatpur. Nepal Med. Coll. J. 13(1): 30-33.

Ahmed, K. and Imran. 2008. Prevalence and antibiogram of uncomplicated lower urinary tract infections in human population of Gilgit, Northern areas of Pakistan. Pakistan J. Zool. 40(4):295-301.

Akinjogunla, O.J. and I.O. Enabulele. 2010. Virulence factors, plasmid profiling and curing analysis of multidrug resistant Staphylcococus aureus and coagulase negative Staphylococcus spp. isolated from patients with acute otitis media. Journal of American science 6(11): 10221033.

Akram, M., M. Shahid and A.U. Khan. 2007. Etiology and antibiotic resistance patterns of community-acquired urinary tract infections in JNMC hospital Aligarh, India. Ann. Clin. Microbiol. Antimicrob. 6: 4.

Alos, J.I., M.G. Serrano and J.L. Gomez-Garces. 2004. Antibiotic resistance of Escherichia coli from community-acquired urinary tract infections in relation to demographic and clinical data. Clin. Microbiol. Infect. 11: 199-203

Alzohairy, M. and H. Khadri. 2011. Frequency and antibiotic susceptibility pattern of uro-pathogens isolated from community and hospital -acquired infections in Saudi Arabia- A prospective case study. Br. J. Med. Res. 1(2): 45-56.

Arjunan, M., A.A. Al-Salamah and M. Amuthan. 2010. Prevalence and antibiotic susceptibility of uropathogens in petients from a rural environment, Tamilnadu. American Journal of Infectious Disease 6(2): 29-33.

Bahadin, J., S.S.H.Teo and S. Methew. 2011. Aetiology of community-acquired urinary tract infection and antimicrobial susceptibility patterns of uropathogens isolated. Singapore Med. J. 52(6): 415-420.

Baral, P., S. Neupane, B.P. Marasini, K.R. Ghimire, B. Lekhak and B. Shrestha. 2012. High prevalence of multidrug resistance in bacterial uropathogens from Kathmandu, Nepal. BMC Res. Notes. 5:38.

Bashar, M.A., M.F. Ahmed, S.R. Rahman and D.J. Gomes. 2009. Distribution and resistance trends of Escherichia coli from urinary tract infection isolated in Dhaka city. Bangladesh J. Med. Sci. 15(2): 93-98.

Bean, D.C., D. Krahe and D.W. Wareham. 2008. Antimicrobial resistance in community and nosocomial Escherichia coli urinary tract isolates, London 2005-2006. Ann. Clin. Microbiol. Antimicrob. 7: 13.

Behroozi, A., M. Rahbar and J.A. Yousefi. 2010. A survey on epidemiology of urinary tract infections and resistance pattern of uropathogens in an Iranian 1000-bed tertiary care hospital. Afr. J .Microbiol. Res. 4(9): 753-756.

Biadglegne, F. and B. Abera. 2009. Antimicrobial resistance of bacterial isolates from urinary tract infections at Felge Hiwot Referral Hospital, Ethiopia. Ethiop. J. Health Dev. 23(3): 236-238.

Bobos, C., C. Hodarnau, D. Terec, L. Feticu, F. Iencica and H. Alina. 2010. Prevalence and susceptibility to chemotherapeutic agents of bacterial species isolated from urinary tract infections. Clujul Medical. 83(1): 69-75.

Chhetri, P.K., S.K. Rai and U.N. Pathak et al. 2001. Retrospective study on urinary tract infection at Nepal Medical College Teaching Hospital, Kathmandu. Nepal Med. Coll. J 3: 83-85.

CLSI. 2011. Performance standards for antimicrobial susceptibility testing; Twenty-first Informational Supplement. CLSI M100-S21:31(1).Clinical and Laboratory Standards Institute, Wayne, PA.

Das, R.N., T.S. Chandrashekhar, H.S. Joshi, M. Gurung, N. Shrestha and P.G. Shivananda. 2006. Frequency and susceptibility profile of pathogens causing urinary tract infections at a tertiary care hospital in Western Nepal. Singapore Med. J. 47(4): 281-285.

DoHS. 2012. Annual report 2010/2011. Department of Health Services (DoHS), Ministry of Health, 
Nepal Journal of Science and Technology Vol. 14, No. 1 (2013) 177-184

Government of Nepal, Teku, Kathmandu, Nepal, $301 \mathrm{pp}$.

Dhakal B.K., B.M. Pokhrel and J. Ahnn. 2002. Microscopic detection of urinary tract infection in Nepalese patients. J. Microbiol. 40(4): 267-273.

Dimitrov, T.S., E.E. Udo, M. Emara, F. Awni and R. Passadilla. 2003. Etiology and antibiotic susceptibility patterns of community-acquired urinary tract infections in a Kuwait hospital. Med Princ Pract. 13(6): 334-339.

Dromigny, J.A., P. Nabeth, A. Juergens-Behr and J.D. Perrier-Gros-Claude. 2005. Risk factors for antibioticresistant Escherichia coli isolated from community-acquired urinary tract infections in Dakar, Senegal. J. Antimicrob. Chemother. 56(1): 236-239.

Ehinmidu J.O. 2003. Antibiotic susceptibility patterns of urine bacterial isolates in Zaria, Nigeria. Trop. J. Pharm. Res. 2(2): 223-228.

Eryilmaz M., M.E. Bozkurt, M. Murat, M.M. Yildiz and A. Akin. 2010. Antimicrobial resistance of urinary Escherichia coli isolates. Trop. J. Pharm. Res. 9(2): 205-209.

Farshad, S., F. Emamghoraishi and A. Japoni. 2010a. Association of virulent genes hly, sfa, cnf-1 and pap with antibiotic sensitivity in Escherichia coli strains isolated from children with community-acquired UTI. Iran Red Crescent Med. J. 12(1): 33-37.

Farshad, S., M. Anvarinejad and A.M. Tavana. 2011. Molecular epidemiology of Escherichia coli strains isolated from children with community acquired urinary tract infection. African Journal of Medical Research 5(26): 4476-4483.

Farshad, S., R. Ranjbar, M. Anvarinejad, M.A. Shahidi and M. Hosseini. 2010b. Emergence of Multi Drug Resistant Strains of Escherichia coli isolated from Urinary Tract Infection. Open Conf. Proc. J. 1: 192196.

Gonzalez, C.M. and A.J. Schaeffer. 1999. Treatment of urinary tract infection: what's old, what's new, and what works. World J. Urol. 17: 372-382.

Hassan, S.A., S.A. Jamal and M. Kamal. 2011. Occurrence of multidrug resistant and ESBL producing E. coli causing urinary tract infections. J. Basic Appl. Sci .7(1): 39-43.

Jadhav, S., A. Hussain, S. Devi, A. Kumar, S. Parveen, N. Gandham, L.H. Wieler, C. Ewers and N. Ahmed. 2011. Virulence characteristics and genetic affinities of multiple resitant uropathogenic Escherichia coli from a semi-urban locality in India. PLoS One 6(3): e18063.

Jamie, W.E., R.K. Edwards and P. Duff. 2002. Antimicrobial susceptibility of gram-negative uropathogens. Infect. Dis. Obstet. Gynecol. 10: 123-126.

Jha, N. and S.K. Bapat. 2005. A study of sensitivity and resistance of pathogenic microorganisms causing UTI in Kathmandu valley. J Med. 3: 123-129.
Kahlmeter, G. 2000. The ECO*SENS Project: a prospective, multinational, multicentre epi-demiological survey of the prevalence and antimicrobial susceptibility of urinary tract pathogens-interim report. J. Antimicrob. Chemother. 46(Suppl A): 15-22.

Karki, A., B.R. Tiwari, and S.B. Pradhan. 2004. Study of bacteria isolated from urinary tract infection and their sensitivity pattern. J. Nepal. Med. Assoc. 43: 200-204

Kattel, H.P., J. Acharya, S.K. Mishra, B.P. Rijal and B.M. Pokhrel. 2008. Bacteriology of urinary tract infection among patients attending Tribhuvan University Teaching Hospital, Kathmandu, Nepal. JNAMLS 9(1): 25-29.

Kausar, Y., S.K. Chunchanur, S.D. Nadagir, L.H. Halesh and M.R. Chandrasekhar. 2009. Virulence factors, serotypes and antimicrobial susepectibility pattern of Escherichia coli in urinary tract infections. Al. Ameen. J. Med. Sci. 2(1): 47-51.

Krumpermann, P.H. 1983. Multiple antibiotics resistance indexing of $E$. coli to identify high risks sources of fecal contamination of foods. App. Environ. Microbiol. 46: $165-170$.

Kumari, N., G. Ghimire, J.K. Magar, T.M. Mohapatra, and A. Rai. 2005. Antibiogram pattern of isolates from UTI cases in Eastern part of Nepal. Nepal Med. Coll. J. 7: 116-118.

Levitt, P.N. 1993. Analysis of pathogens isolated from urinary tract infection in Barbados. West Indi. Med. J. 42: $72-76$.

Manjunath, G.N., R. Prakash, A. Vamseedhar and K. Shetty. 2011. Changing trends in the spectrum of antimicrobial drug resistance pattern of uropathogens isolated from hospital and community patients with urinary tract infections in Tumkur and Bangalore. Int. J. Biol. Med. Res. 2(2): 504-507.

Mazzulli ,T., M. Skulnick, G. Small, W. Marshall, D.J. Hoban, G.G. Zhanel, S. Finn and D.E. Low 2001. Susceptibility of community Gram-negative urinary tract isolates to mecillinam and other oral agents. Can. J. Infect. Dis. 12(5): 289-292.

Miller, L.G. and A.W. Tang. 2004. Treatment of uncomplicated urinary tract infections in an era of increasing antimicrobial resistance. Mayo Clin. Proc. 79: 1048-1054.

Moniri, R., A. Khorshidi, and H. Akbari. 2003. Emergence of multidrug resistant strains of Escherichia coli isolated from urinary tract infection. Iranian J. Publ. Health 32(4): 42-46.

Moyo, S.J., S. Aboud, Kasubi M, Lyamuya E.F. and S.Y. Maselle. 2010. Antimicrobial resistance among procedures and non-producers of extended spectrum beta-lactamase in urinary isolates at a tertiary hospital in Tanzania. BMC Research Notes 3: 348.

Nicolle, L., P.A.M. Anderson, J. Conly, T.C. Mainprize, J. Meuser, J.C. Nickel, V.M. Senikas and G.G. Zhanel. 
2006. Uncomplicated urinary tract infection in women. Can. Fam. Physician. 52(5): 612-618.

Okonko, I.O., O.B. Donbraye and L.A. Ijandipe. 2009. Antibiotic sensitivity and resistance patterns of uropathogens to nitrofurantoin and nalidixic acid in pregnant women with urinary tract infections in Inbadan, Nigeria. Middle East Journal of Scientific Research 4(2):105-109.

Okonofua, E.E. and B.N. Okonofu. 1989. Incidence and pattern of asymptomatic bacteriuria of pregnancies in Nigerian women. Nig. Med. Pract. 17: 354-358.

Omigie, O., L. Okoror, P. Umolu, and I.G. Kuuh. 2009. Increasing resistance to quinolones: A four-year prospective study of urinary tract infection pathogen. Int. J. Gen. Med. 2:171-175.

Orrett, F.A., and G.K. Davis. 2006. A comparison of the antimicrobial susceptibility profile of urinary pathogens for the years 1999 and 2003. West Indian Med. J. 55: 95-99.

Rafique, S., A. Mehmood, M. Qayyum and A.B. Qazilbash. 2002. Prevalence patterns of community-based and nosocomial urinary tract infection caused by Escherichia coli. Pakistan Journal of Biological Sciences 5(4): 494-496.

Rai, G.K., H.C. Upreti, S.K. Rai, K.P. Shah and R.M. Shrestha. 2008. Causative agents of urinary tract infections in children and their antibiotic sensitivity pattern: a hospital based study. Nepal Med. Coll. J. 10(2): 86-90.

Raksha, R., H. Srinivasa, and R.S. Macaden. 2003. Occurrence and characterization of uropathogenic Escherichia coli in urinary tract infections. Indian J. Med. Microbiol. 21(2): 102-107.

Ramesh, N., C.S. Sumathi, V. Balasubramanian, K.R. Palaniappan and V.R. Kannan. 2008. Urinary tract infection and antimicrobial susceptibility pattern of extended spectrum of beta lactamase producing clinical isolates. Advan Biol Res. 2(5-6): 78-82.

Rashedmarandi, F., M. Rahnamayefarzami, M. Saremi and R. Sabouri 2008. A survey on urinary pathogens and their antimicrobial susceptibility among patients with significant bacteriuria. Iran J Patho.l 4: 191-196.

Raza, S., S. Pandey and C.P. Bhatt. 2011. Microbiological analysis of the urine isolates in Kathmandu Medical College Teaching Hospital, Kathmandu, Nepal. 2011. Kathmandu Univ. Med. J. 36(4): 295-297.

Rijal, A., G. Ghimire, K. Gautam and A. Barakoti. 2012. Antibiotic susceptibility of organism causing urinary tract infection in patients presenting to a teaching hospital. J. Nepal Health Res. Counc. 10(20): 24-27.

Sahm, D.F., C. Thornsberry, D.C. Mayfield, M.E. Jones and J.A. Karlowsky. 2001. Multidrug resistant urinary tract isolates of Escherichia coli: Prevalence and patients demographics in the United States in 2000. Antimicrob. Agents Chemother. 45(5): 1402-1406.

Saleh, A.A., S.S. Ahmed, M. Ahmed, A.N.I. Sattar and Md.R.A. Miah 2009. Changing trends in uropathogens and their antimicrobial sensitivity pattern. Bangladesh J Med Microbiol. 3(1): 9-12.

Sharma, A., S. Shrestha, S. Upadhyay and P. Rijal 2011. Clinical and bacteriological profile of urinary tract infection in children at Nepal Medical College Teaching Hospital. Nepal Med. Coll. J. 13(1): 24-26.

Shrestha P., S. Malla, S. Basnvat, S. Dumre, B. Upadhaya and S. Lamichhane. 2007. Antimicrobial resistance pattern of bacterial isolates causing urinary tract infection. J. Nepal Health Res. Counc. 5(2): 49-54.

Tambekar, D.H., D.V. Dhanorkar, S.R. Gulhane, V.K. Khandelwal and M.N. Dudhane. 2006. Antibacterial susceptibility of some urinary tract pathogens to commonly used antibiotic. Afr. J. Biotechnol. 5(17): 1562-1565. 
Nepal Journal of Science and Technology Vol. 14, No. 1 (2013) 177-184 